\title{
Intelligent Production of Quality Apparels
}

\author{
R.C. Michelini and R.P. Razzoli*
}

University of Genova, DIME, Dipartimento di Ingegneria Meccanica, Energetica, Gestionale e dei Trasporti, Italy

\begin{abstract}
Example applications are developed, covering typical sections of garments manufacturing and addressing robotic solutions in three different contexts. The first deals with the fabric storing and sorting sections. The case aims at the integrated management of cloth data (included each bolt failure maps), so that the work-plans setting progresses according to optimal schedules, without surprises of defective parts to be replaced. The change is made possible after redesign of fabric warehousing, with higher automation, in order that all pertinent data on cloth quality are monitored, shared, exploited and up-dated. The second one addresses the laying and cutting sections, already fully operated by unmanned mode. The upgrading looks at knowledge intensive setting, for on-process management of discontinuities, making easy the combined-mode (batch and one-of-a-kind) schedules to expand plant flexibility, joined to early removal of defective parts (using fabric fault maps). The development specifically requires re-thinking the enterprise policy, with minor changes of the computer interfaces, but proper resetting of the shop information system. The third context differs, as we refer to assembly and sewing sections, still, mainly operated by front-end workers. It should be said that the setting of fully automatic sewing cells has been conceived and actual fixtures exist for the mass production of steady specialised articles; yet, this is hardly consistent with economy of scope; a different implementation is outlined, with front-end workers and shared information steering duty-cycle changes. Again, proper improvements are obtained, with benefits requesting little investments in hardware, rather than work-plans up-dating depending on the process progression.
\end{abstract}

Keywords: Robotics in Textiles, Customised Clothes, Integrated Automation, Materials vs. Information Flows.

\section{INTRODUCTION}

Robot concepts have increasing importance in clothing manufacturing, enabling the information flow (from ideation and strategic planning, to shop ruling, flexibility decision and output control), in parallel of the material flow (from fabrics and supplies, to laying, cutting, assembly and sewing of outfits, dresses and apparel). The integration options allow devising customer-driven product mixes within a quickresponse time frame, by organised high quality and reliability. Until now, most manufacturers prefer manual work-cycles, moving labour intensive tasks to low-wage countries, with material flow fragmentation and overseeing discontinuity. Then, the creation of high quality clothing is viewed as handicraft; innovation limits at assisting skilled people at the ideation phases, with only concern in enhancing product value creation, from the customer's viewpoint.

The spread apart of the two flows is extant way of clothing manufacturers. The fashion industry chiefly operates on a recurrent basis, with several months of leadtime in planning each season. The manufacturers try to anticipate tastes and whims of buyers; they attempt to stock sufficient quantities of garments, to meet the expected demand. This may not be economical: both under and over estimating demand has a cost. Sadly, little can be done to

\footnotetext{
*Address correspondence to this author at the University of Genova, DIME, Dipartimento di Ingegneria Meccanica, Energetica, Gestionale e dei Trasporti, Italy; Tel: +39 0103532844; Fax: +39 0103532844;

E-mail: razzoli@dimec.unige.it
}

recover from poor estimates, but an appropriate form of flexible automation and factory intelligence offers the potential for effectively handling market driven orders on a more real-time basis, providing improvements in efficiency, as well as providing improvements in efficiency in the production of customised garments. From the present scheduling situation, the prospected changes move to flexible automation, enabling fabrication agendas, mixing batch and one-of-a-kind garments. This will drastically change the current habits.

The joint batch and one-of-a-kind processing shall cover the typical sequence, Fig. (1):

- cloth bolts warehousing and selective materials delivery;

- fabric laying and cutting with pieces buffering and sorting;

- sewing sections with feeding and material logistics;

- pressing sections and items final finishing;

- quality checking, packing and garments storing;

- $\quad$ supervision for retail supply and marketing.

The Fig. (1) depicts this mixed-mode planning. The up grading of traditional manufacturing areas, such as textile and clothing, is very important. We need exploring distributed intelligence styles, including theories already termed in the literature, [1-5]. Changes must be viewed weighting returns, and understanding that, today, near a revolution is necessary, to win back world-wide 


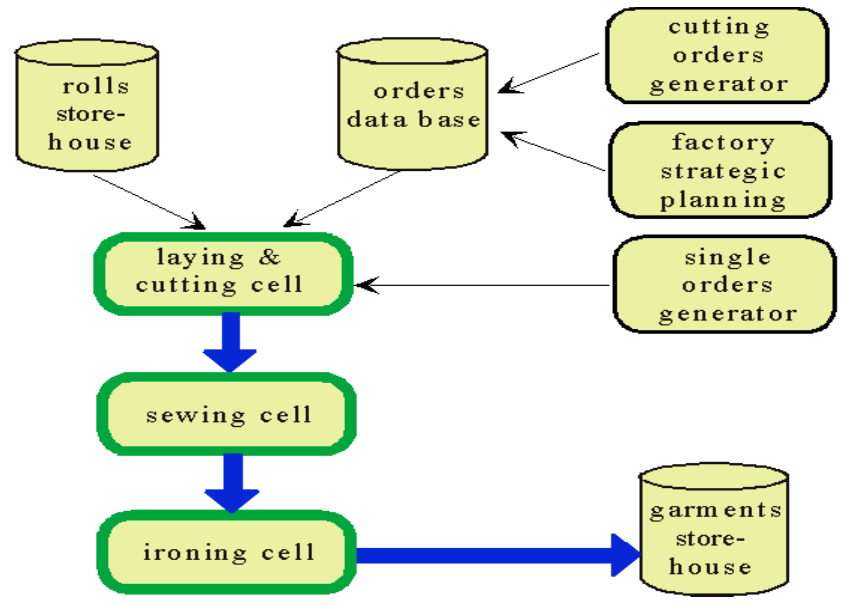

Fig. (1). Flow-chart for batch-and-customised manufacturing.

effectiveness. In Fig. (1), the production of <assorted batches〉 and «one-of-a-kind schedules merges, looking at:

- efficiency planning, aiming at the delivery of shrewdly balanced supply flows;

- time-to-market provision, assuring the precise execution of customised indents.

The twin goal is possible and can assure return on investment, when aimed at quality apparels. The challenge is alternative to productive break-up and out-sourcing. Developed countries are interested in preserving life-quality, creating jobs for the benefit of value-generating businesses, having products and processes compatible with the environment. The paper offers cues in that direction.

\section{EFFICIENCY FROM QUALITY}

The manufacture of high-quality clothing benefits from advanced design ideas and intelligent production technology. Items are sold with high added value, driven by consumer satisfaction; marketing is global in scope, with rapidly changing fashion trends. Buyers are right away aware of quality, reliability and value/price ratio; manufacturers are confronted by demanding tasks, with pressure on prices, new fashions, customisation, wide product mix, quick response with dependable delivery dates, and the likes. Economies of scope can replace economies of scale, following typical patterns, including flexible specialisation, lean engineering, company-wide quality, and continuous product improvement $[6,7]$. Creation of high-quality clothing includes skilled labour at the ideation phases, and planned concern to keep critical jobs inside the factory. The outside allocation of labour intensive cycles may be pursued for prompt returns. On midterm to long term horizons, a rather different strategy needs to be considered, based on a series of steps, viz.:

- balanced production plans on the strategic horizons, entailing the total available facilities;

- maintenance monitoring of the work-cycles, for steady quality and zero-defect deliveries;

- versatility integrated control-and-management, for just-in-time programmed supply chains;

- pace-wise product-and-process upgrading, with transparency of intermediate attainments.
The scope alternative, focusing <quality>, addresses technology to improve efficiency, aiming at enhancing the design/manufacturing of outstanding products, with attention on information flow, material procurement, marketing orders, etc., to promote innovative ideas, by managing process intelligence [8, 9]. Besides, automation in the processing of clothing presents some unique problems compared to other industrial areas, due to rather peculiar traits:

- the human-friendly base work-schedules, chiefly, without dangerous tasks;

- the wide range of skills, rooted in combined (rather than elemental) duties;

- the personal commitment required for the training of the worker's expertise;

- the limp behaviour of fabric and other stuffs, concerned by the processing;

- the creative ideation/forming of 3D shapes, by the warping of $2 \mathrm{D}$ pieces;

- $\quad$ the dexterity preparation, guided by well-established acts and know-how.

The evolution of textile and clothing industries on automation requires explicit areas-adapted issues. There is no doubt that the mechanisation with ingenious devices developed in these areas (looms with task-driven commands, lace and embroidery needle-working machines, etc.), since front-end rigs provide mass-production by automated setting. Yet, recently, flexible manufacture remains at the outboard, with little invention at front-end fixtures and low data integration from process and product monitoring.

The choice deserves notice, as it represents a peculiarity, compared to extant trends that modify apparel business in the affluent countries, with awkward outcomes, e.g., the habit of looking for short-use thrown-away items, without concern on quality and durability. We alternatively have changes, aiming at robot technologies based on the suited exploitation of process information, merged into the conventional material flow $[10,11]$.

The investigation recalls a series of projects specially concerned by quality apparels, where the value chain offered by industrial planning can out-perform handicraft production 
Composition - Number of ends and picks - Weave

Colour fastness to light, to perspiration, to dry cleaning, to washing, to dry pressing/ironing, to rubbing, to dry spotting, etc.

Breaking strength and elongation (longitudinal/transversal) - Tear endurance - Breaking strength (grab method)

Resistance to abrasion, to pilling, to snagging, to dry ravelling, etc.

Seam slippage - Crease recovery - Dimensional stability to washing, to dry cleaning, etc.

Yarn defects: broken threats, curling, twin top yarn, uneven glossiness, knots, etc.

Warp defects: barred weaving, furrows, stack, faulty warping, chopped-off warp, etc.

Woof defects: lacking yarn, wrong weft, stretched/winding thread, fake reach, etc.

Flaws after dying, printing, finishing: unevenness, stains, blurs, creases, slobbers, etc.

Selvedge flaws: folded listing, holed selvage, damaged boundaries, etc.

Extra deficiencies: skew slant, drawing asymmetry, holes, inclusions, uneven width, etc.

Fig. (2). Typical features/defects of woven fabrics.

for steadiness and reliability. For explanatory purposes, three manufacture sections are addressed:

$>$ the storage/retrieval processes of fabric from the warehouse in preparation for manufacture of varying mixes of garments, as for type, model, size, etc.: efficiency depends on exploiting the existing information, on on-line managing the fabric qualitydata, by purposely developed front-end handling equipment.

$>$ the laying and cutting processes of garment components, today, already accomplished by programmable machines, i.e., noteworthy fixed automation rigs: attention is on intelligence for online adaptive flexibility; an example is considered to show productivity effects, when one-of-a-kind and batch products are simultaneously processed.

$>$ the assembly and sewing processes of garments, mainly deferred to hybrid stands, with front-end operators interfaced to automation heads: scope (vs. scale) economy ideas are analysed, so a benchmarking strategy allows evaluating the shop effectiveness by on-process managing the distributed intelligence.

The relevant role is played by the enterprise information systems and tied acquisition, storing and retrieval modules. These topics are today properly appreciated; the developed hardware and software tools are established technology and little needs to be added to explore their transfer to textile and clothing industries. The investigation is, therefore, more specifically interested to the tools supporting knowledge build-up and decision making, in connection with the considered business. Computer simulation is introduced as important aid, for setting adaptive govern rules on the automation of the manufacturing process. We distinguish several types of aids. Resort to digital mock-ups and to virtual reality testing is fundamental engineering, to accomplish lifecycle tests on (digital) prototypes, so that the actual behaviour of the allotted facilities is properly assessed under the scenario of the forecast engagements.

Computer simulation is off-process aid at the design phase, to find out the most effective lay-out for given engineering specifications. Intelligent automation, moreover, confronts with varying duty layouts, and conflicts with the concept of optimal setting. On-process computer simulation, then, is current decision support to up-date resource allocation and to adapt fabrication agendas, according to economy of scope rules, with account of time horizons and planning ranges.

The three example developments cover the different front-end automation options. They, quite properly, represent reference cases, when the attention is focused on return on investment in material processing facilities. The present study reconsider the problems widening the focus on the joint material-and-information flows management. The situation further changes, if quality clothes are considered, where the value chain will rise in terms of customised quality reliability. The upgrading does not face steady ends: at times, enhanced material flow concern is winning step to help data flow integration; sometimes, the automation level is already fully developed, and integration is straightforward; some other times, return on investment for further automation is questionable, and data flow integration could resort to inclusion of front-end workers. All in all, the developments provide a general view of the issues offered by distributed intelligence in the shop-floor organisation, with varying automation ranges.

\section{FABRIC WAREHOUSING AND HANDLING}

At first, the intelligent factory addresses <materials-andinformation> joined flow: combined inputs grant deliveries with optimised value. The fabric supply is critical [12]. It shall handle the pertinent data on the warehouse inventory, the cloth types and lengths, the procurement policy, etc., for inventory and purchasing information; for production plans, special data for the stored rolls are required, including handling rules, characteristic features, fault maps and classification, etc., Fig. (2). These data provide the background knowledge for front-end operations. They also provide the foreground information, to implement combined control and management logistics.

The process of rating defects, conventionally, distinguishes:

- $\quad$ slight faultiness: defects do not affect, due to location or low probability of being noticed;

- heavy faultiness: the defects are apparent, compromising the completed garments' quality;

- fatal faultiness: the defective piece of cloth would make a finished item useless or unsafe.

The cloth characteristics are object of established standards and measurement techniques. The provision avails of bolts, with annexed maps of the qualifying features and defects. The maps are input data, mainly, in view: 

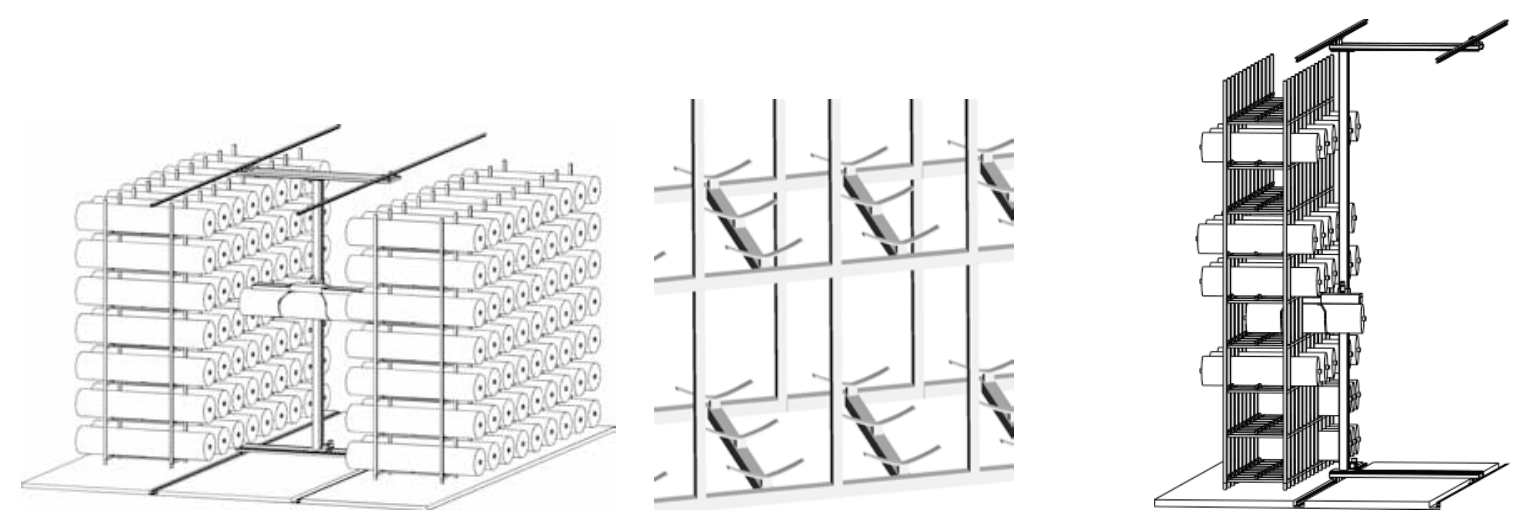

Fig. (3). Fabric warehouse: layout, supporting frame, pick/place rig.
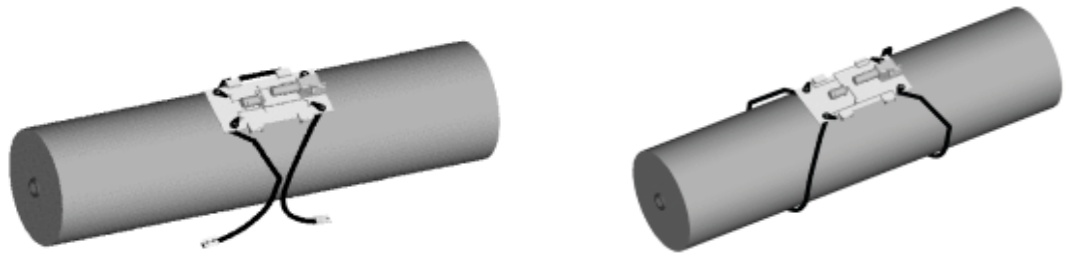

(a)
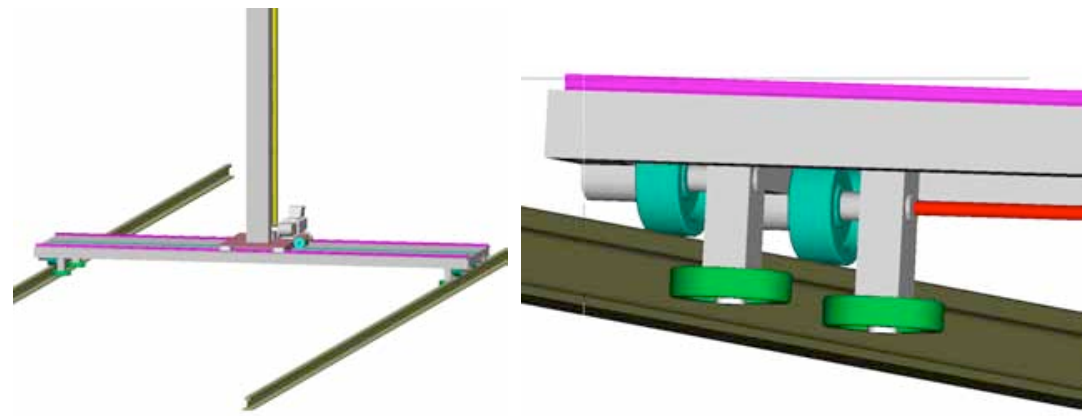

(b)

Fig. (4). Roll grasping: engagement/latching; tower translation and roller details.

- to schedule the cutting policies, to manage neutralising the slight faultiness;

- $\quad$ to plan extra-length to duplicate the piece cutting with heavy faultiness bolts;

- to eliminate, from the concerned bolt, the complete fatal faultiness portions.

The faultiness handling goes together with production scheduling. The manufacture of quality clothing, basically, follows two guidelines for a successful return on investment:

- for ready-made clothing, production efficiency is main request: the market demand for a given span should match the season's total number of clothes, by tactical horizon adapted productivity;

- market-driven manufacturing should be available for quick response opportunities: the one-of-a-kind personalised items can be efficiently managed in process, within an operational time frame.

Fabric warehousing and retrieval technology are fair contribution in both cases. In ready-made production, efficiency and lean manufacturing concepts play a major role in scrap reduction and production effectiveness. In terms of flexible manufacturing for custom made clothing, the retrieval process is especially important to allow varying items mix or limited production runs to be inserted as case arises, without relevant downgrading the normal production schedule.

The Fig. (3) shows the basic elements of the fabric warehousing, exemplifying the roll storing frame and the pick/place rig: this has a suited gripper, Fig. (4), with twin sliding arms, carried by a mobile tower. This way, each single bolt has fully automatized handling and management.

The computer-aided planning avails of a process simulation code, Fig. (5), describing the fabric flow, according to each especially detailed service. The controller performs the scheduling and coordination, minimising the waste and supplying laying and cutting data for further processing.

The simulation code supplies off-process anticipation of the planned schedules, to test the actual achievements. Later, it permits, Fig. (6), viewing the individual production course, to verify the nested lay-out, with the pertinent details (e.g., location of defects, if any).

The duty monitoring provides real-time data and statistical records. For example purpose, we consider the opportunity of mixing together batch orders and one-of-a- 


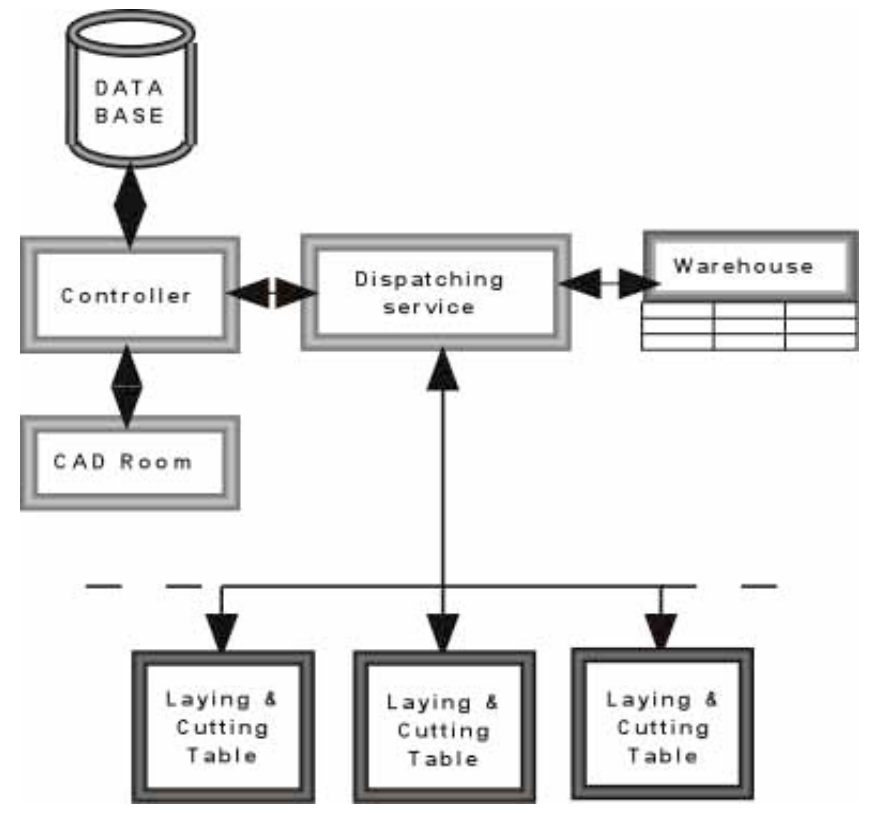

Fig. (5). Architecture of the RSS-SIFIP simulation code.

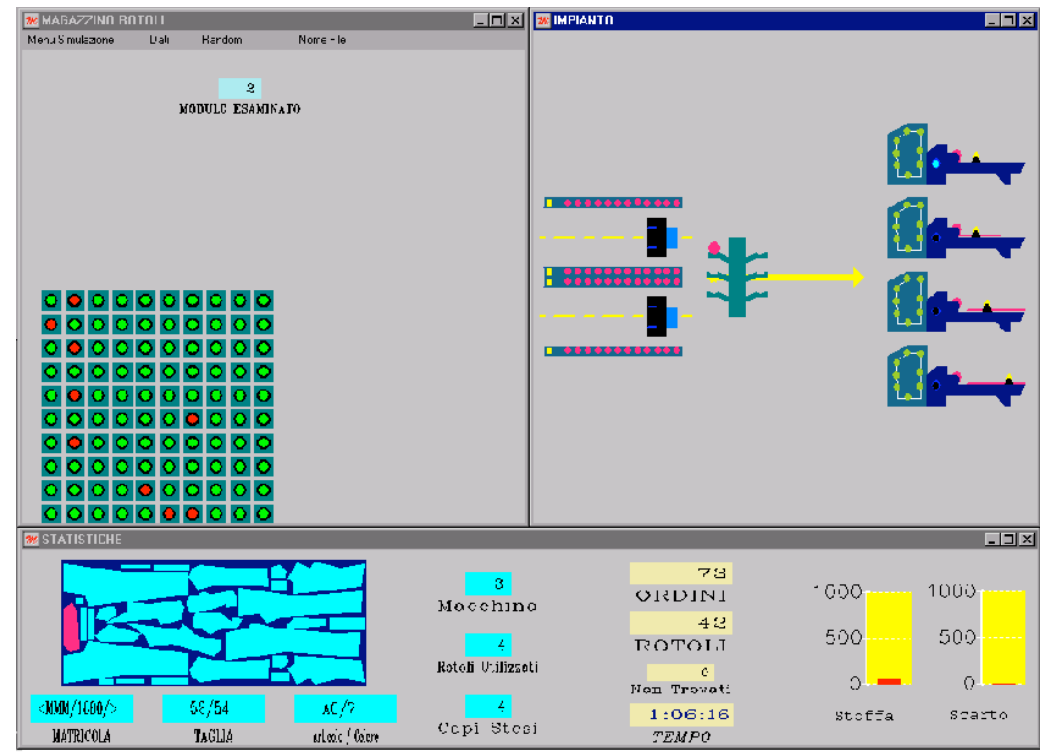

Fig. (6). Example RSS-SIFIP window for the process monitoring.

kind items (including replacements for flawed garment parts, as just mentioned). During on-line operations, the part layout choice and knowledge of the defect maps suggest specialnesting ideas to grant clothing quality or, alternatively, a subset of garment parts could result with excessive faults. The study, then, needs to be carried together with the characterisation of the laying/cutting section.

\section{FABRIC LAYING AND CUTTING}

The automation of the warehousing and retrieval process is logical start to establish a distributed intelligence organisation. The laying and cutting are already done into fully automatic stands, by long tables allowing unwrapping several superposed layers, where the 'windows' distinguish size or model of identical parts assortments. Yield depends on the number of layers aimed at mass production. With customised items, the window deals with a single layer, to be, as well, exploited to manage heavy faultiness; changes in product mix affect the <to automate> strategic decision: instead of throughput and due-time wavers, by optimal topdown schedules, operation decision aims at customised garments (to face bottom-up requests), still preserving the structured frames of industrial production, with fault management capabilities.

Concluding the example fault management discussion, we address an extra-length window. The twice cut parts appear marked-up on the display and replacement pieces would be separately cut from a single of the same fabric, Fig. (7).

The simultaneous management of one-of-a-kind items is possible, on condition that the laying tables accept free spans for out-of-batch articles. This way, it is easy to include extra items out of the original production plans straight in real time, or (e.g. when the desired fabric is temporarily not available), pushing the changes to a later batch. The adaptive planning adjustments, needed to allow such flexibility, can 

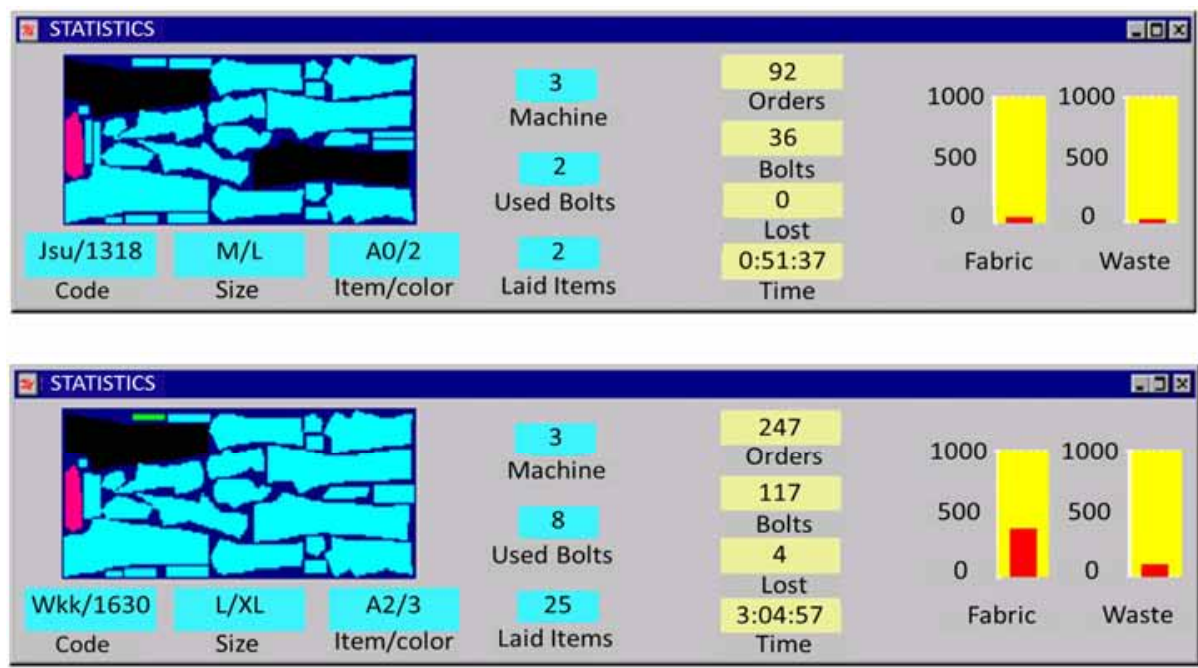

Fig. (7). Windows for the management of defective parts.

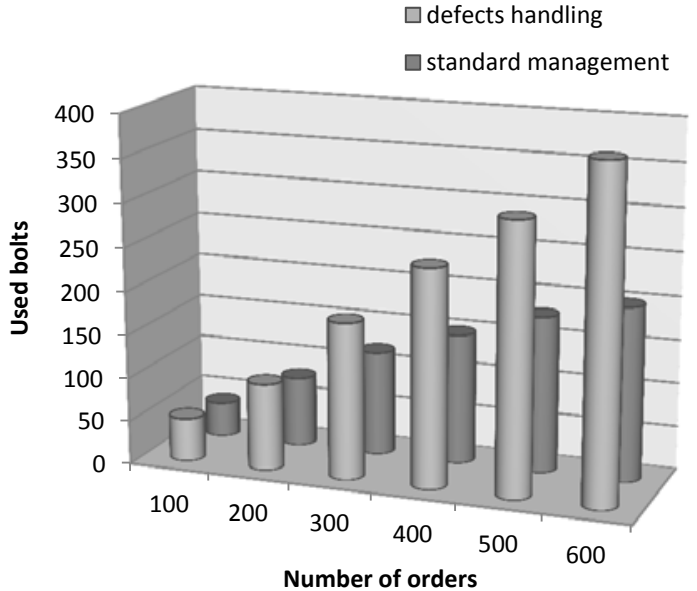

Fig. (8). Handled bolts in case of on-process management of defected parts.

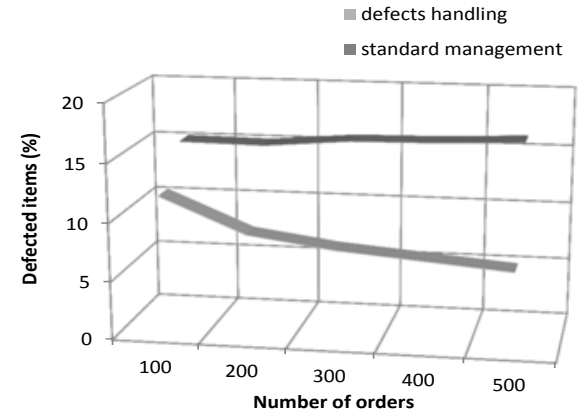

(a)

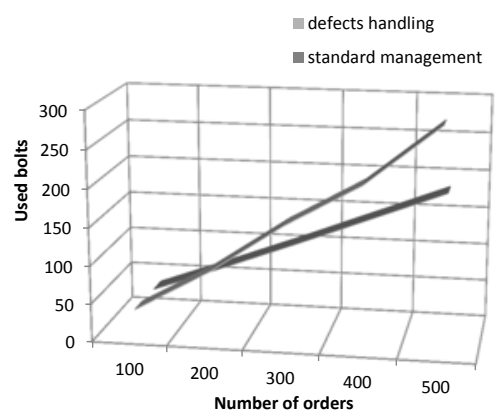

(b)

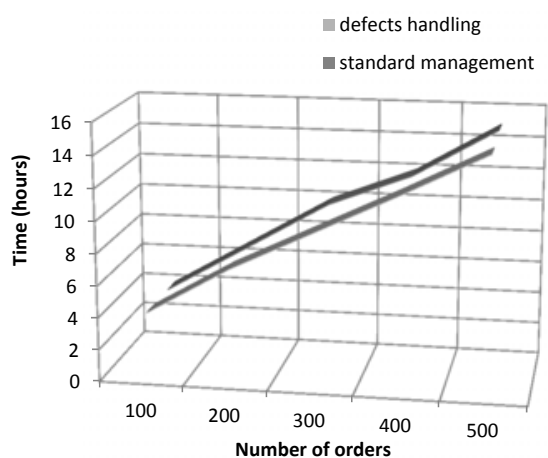

(c)

Fig. (9). Sample results: (a) defected items, (b) used bolts, (c) elapsed time vs. order number.

be provided by interaction with on-line operators or by an expert module, endowed of rule-based decision logic.

The example case data are: the warehouse section consists of four scaffolds with 10x10 frames, 400 total bolts and 20 equally distributed fabric types; the initial roll length ranges from 18 to $20 \mathrm{~m}$, with (duly mapped) 5 to 15 significant faults; the mix of garments requires a set number of cutting spans on the layout table. Depending on the batch size for the garments, the fabric laying process considering defect-mapping shows a considerable waste reduction, Fig. (8), compared to the fit post-cutting recognition of nonusable pieces. The in-process use of fabric data needs the handling of a larger number of bolts, Fig. (9), but this is balanced by the fact that later handling (of the same bolts) for cutting replacement pieces is avoided, also, removing any time-delay. In this case, the optimal bolt search turns to be simple due to a large number of choices. 


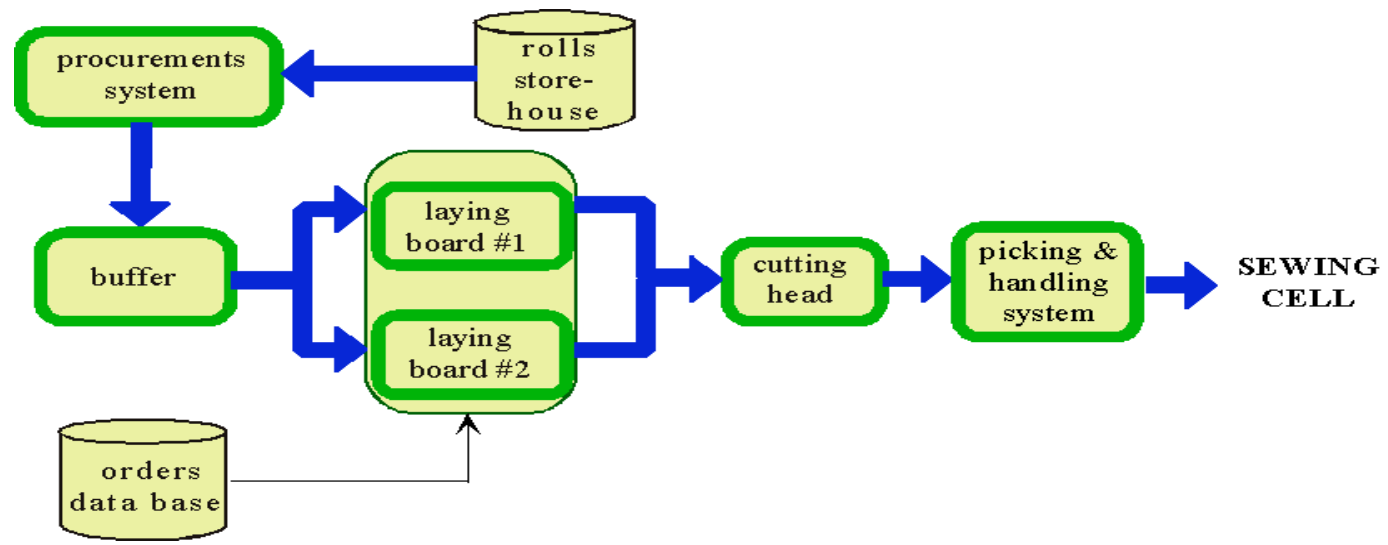

Fig. (10). Architecture of the LCC-SIFIP simulation code.

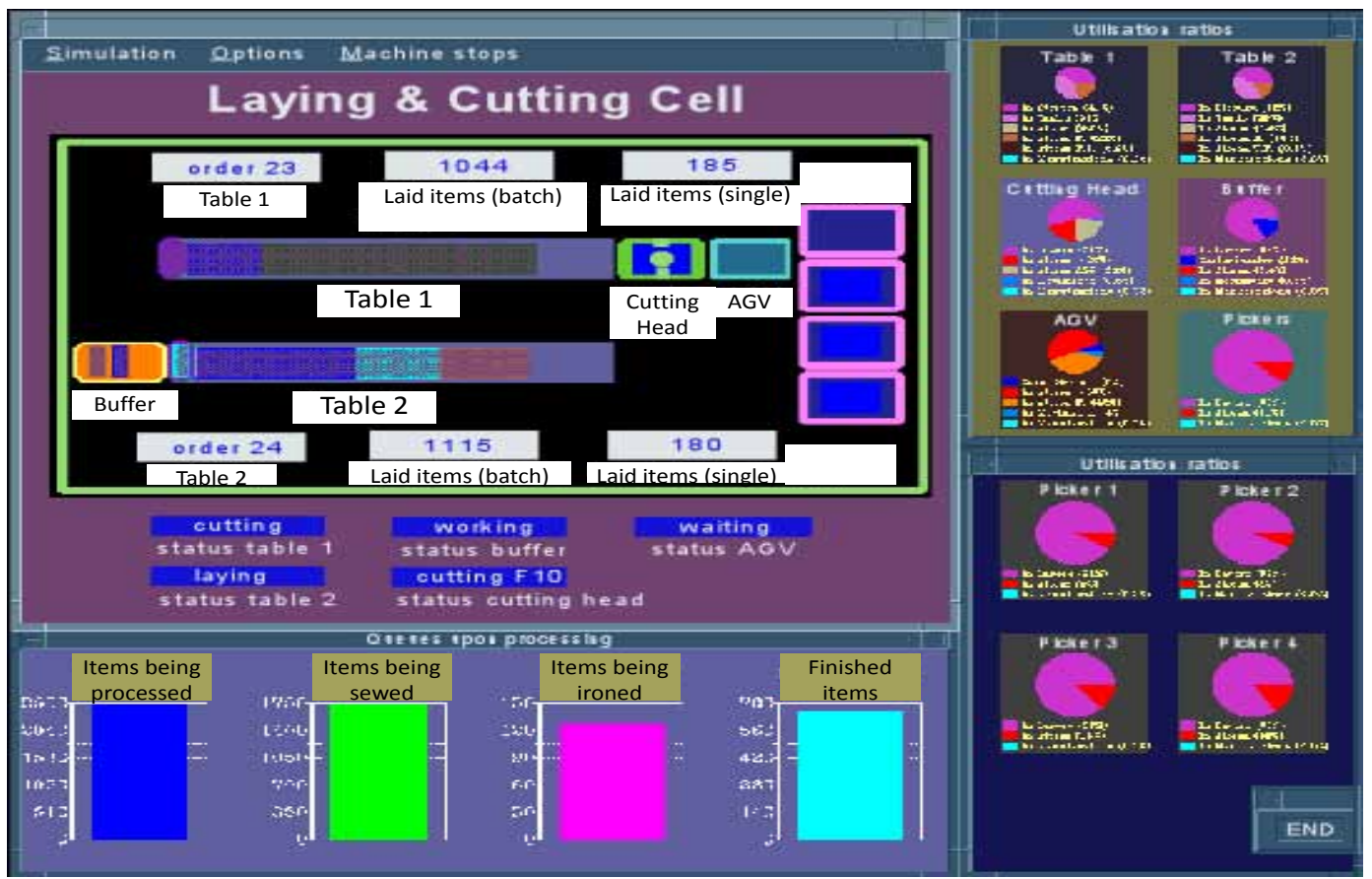

Fig. (11). Example LCC-SIFIP display for the process monitoring.

The quality control is exemplary case where automation provides critical support for production certification. The innovation of, both, material and information allows including:

- flexible/versatile cloth warehouse, with effective database storing and management capabilities;

- shop logistic service for fabric housing and dispatching, by on-process intelligent decision logic.

These changes need to be investigated in terms of the expected returns. Simulation studies are dominant help, to compare competing alternatives referring to actual production contexts and to provide explanatory checks with training purport related to sets of feasible implementations.

The laying/cutting section is key step, to exploit. The facility, Fig. (10), receives information from the order database for the nested cutting, obtains fabric from the warehouse through a buffer, and delivers cut garment parts to the sewing cells. The production flow needs to be analysed, and the functional description shall be detailed for computer simulation. The section is directly interfaced to bolt handling and dispatching facilities, and shares the cloth data.

The example code of Fig. (10) deals with two unwrapping tables, alternatively feeding a cutting station. The display is surrounded by updated information on the production flow and on the utilisation ratio of the allotted resources, Fig. (11).

This information can be exploited to permit the effective interlaced processing of:

- $\quad$ sorted batches, over tactical spans, with delivery to decentralised special shops;

- one-of-a-kind items, to yield quick-response issues, processed in the same shop.

Planning for combined-mode operations (batch plus oneof-a-kind) has critical middle phase at fabric cutting, where automation has wide acceptance and investment return is simply related to productivity. 


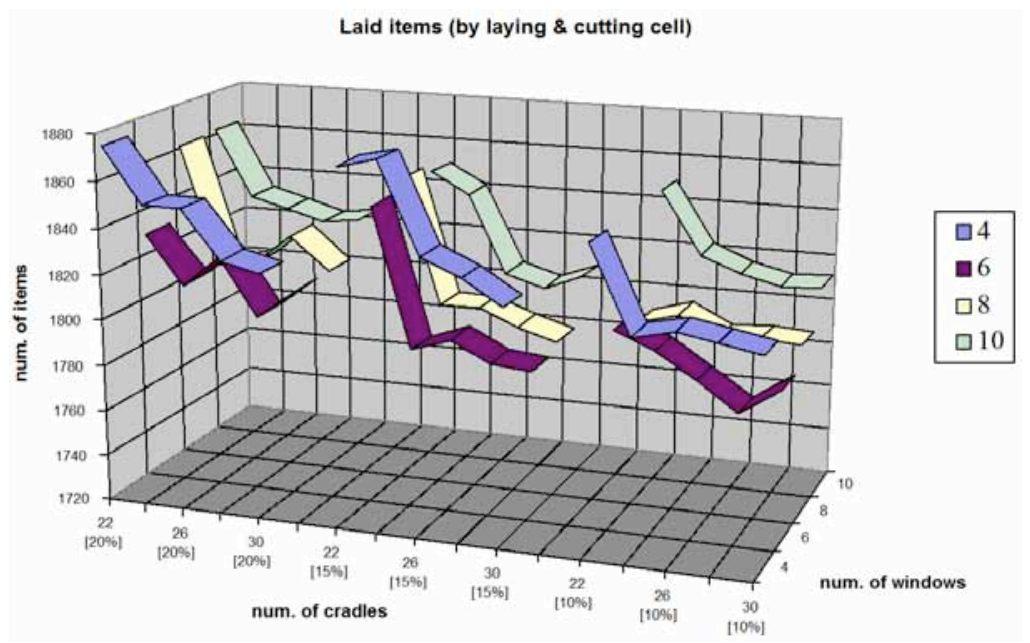

(c)

Fig. (13). Example work-loads of: (a) buffer, (b) cutting head, (c) laid items.

\# facility behavioural model: relational description of the resources dynamics and of the decisions manifold, actually specifying the real plant evolution.

\# facility performance evaluation: factual metrics, measuring the return on investment by referring to varying product mixes and actual production plans.

\# facility simulation software: computer-aids supplying virtual reality miming of competing resource lay-outs and work-programs.

\# facility expert governor: integrated control-and-management units, enabling adaptive schedules depending on the work-in-progress mixes

Fig. (14). Multiple step procedure to appraise flexibility effects in manufacture.

The Fig. (12) gives a view of the cutting tables, with the lay-out of the piled-up pieces, ready for striping and collection in sorted packages to be forwarded to the sewing sections. To understand the actual effectiveness of the onprogress fabrication agenda, the display provides the statistics by current and accumulated figures.

The <intelligent> setting addresses possible benefits e.g., Fig. (13):

- at the organisational range: to select product mix variability under process-attuned managers;

- at the coordination range: to optimise production agendas, under decentralised controllers;

- at the operational range: to deal with on-process discontinuities, under real-time supervisors.

The productivity of the layout and cutting cell depends on many factors. Fig. (13) shows links on current batch size, number of reserved roll places for the feeding buffer (cradles) and also on the number of extra layout windows, added to the regular format. Crafty expedients (on-line resort to fabric defect maps, combined-mode batch/single processing, etc.) are requisites of intelligent manufacture; benefits depend on a large number of cross-related facts, conditioned by material and data flows, whose efficiency critically depends on automatic processing.

\section{SEWING SECTION MIXING}

The sewing of garments is critical step in clothing manufacture. The stage tends to be routine and labour intensive; it is responsible for the productive break-up to low wage countries. The sewing section (of already assorted pieces) may be considered for operations at decentralised locations. On the other hand, the sewing process is a prime candidate for intelligence sharing and it is especially desirable to operate it locally, for the manufacture of customised garments, when quality data are specifically addressed. Since long-term business success is critical goal, balancing added value and cost reduction against decentralisation and preserving high standing products under direct local control (e.g. by sewing supervision and adaptive scheduling) needs to be considered as important chance.

To that purpose, the organisation requirements are reviewed, with aims at methods better suited for on-process improvements. The attention on testing is deepened for, mainly, two reasons: the on-line request of manual operations needs focusing on uncertainty and heuristics, further to automation sharpness; the on-process decision cycle requests real-time govern modules acting with intensive information. A multiple procedure, Fig. (14), is useful preparation of the scheduled facilities.

Customised high quality products are less affected by market saturation, but must reach interested buyers with right price and timing. Workplace organisation shall aim at distributed commitment and cooperative rewards. Data management systems require ready access to economical (finance, accounting), technical (order processing) and operation (master plan) aspects of the sales policy (item, quality, price, due-date, etc.). The evolution of this process is related to the concurrence of material and data flow, with 
Forward chaining, to try deductive checks and to look after the most plausible antecedents within the current data-base.

Backward chaining, to compare all the rules, whose consequent assigns the given value to the considered variable.

Work-space activation, to execute the rules of a work-space, whose antecedents are have been initially set.

Occurrence detection, to run rules with antecedents having a given, whenever specified, occurrence, as soon as this happens.

Recurrent scanning, to periodically or at fixed thresholds repeat the class of properly labelled rules.

Detail focusing, to invoke all the rules that contain a purposeful object or object class.

Fig. (15). Example decision management logic of the SCX-SIFIP code.

\begin{tabular}{l}
\hline Arrival of the shuttle and loading the buffer input with the pallet carrying the assorted parts. \\
\hline Acknowledgement of work schedules. \\
\hline Cycle consistency and transfer of the given pallet to the buffer. \\
\hline Check of the sewing heads correct setting (or reprogramming at a new product mix). \\
\hline (Manual) pieces handling and positioning (with the help of special fixtures). \\
\hline Junction of the pieces by (automatic) sewing operations and items back. \\
\hline Handling. \\
\hline Forwarding the pallet to the next work station and repeating the job as programmed. \\
\hline Loading the buffer output with the finished items pallet (and request of new shuttles). \\
\hline Removal of the pallet (dispatching policy can be tuned to deal with a set of pallets). \\
\hline
\end{tabular}

Fig. (16). Typical operation sequence of robot assisted sewing cells.

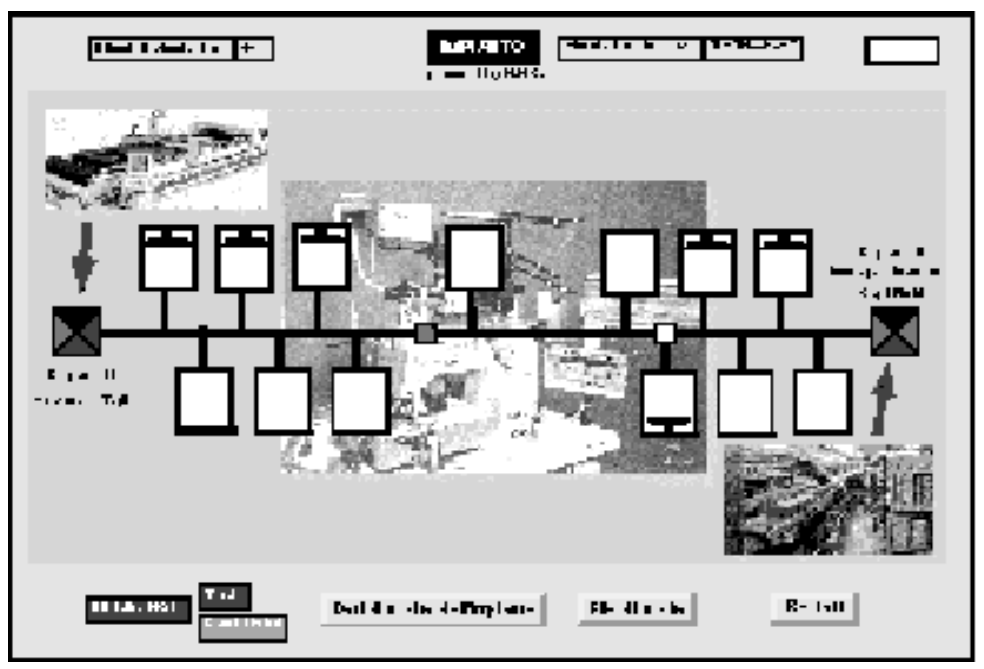

Fig. (17). Example robot assisted sewing section layout.

changes in both ones, driven by the control flow. Economy of scope enables continuous adaptation of current tasks and decision support, tuned to business achievements. Clearly, this upgrading rationale stems from the ability to quantitatively assess the on-going process and issues of the considered changes.

In such frame, the SCX-SIFIP package is built incorporating G2 language features, to fulfil on-process interfacing through rule-based govern modules with realtime ability, covering a wide set of production scenarios, by rule-invoking abilities, Fig. (15).

A case application is developed, exemplifying the expert simulation potentials. The resources of a standard processing cell include the: - set of sewing heads; - I/O buffer; • carrying pallets; $\bullet$ production batches; $\bullet$ product mixes; $\bullet$ inprocess operator; $\bullet$ supplying station (laying/cutting section); - delivery station (finishing section); • dispatching service; • intermediate stores; - quality control set-up. A few comments recall only on the main functional prerequisites. Each cell is fed by a shuttle (on a rail) and possesses a buffer. An automatic loading/unloading rig positions the individual pallets. The operator, placed in front of the (turn by turn) pre-set sewing head, is charged to handle the pieces to be joined. The sewing and quality modules acknowledge these ones and data are displayed, so that the operator follows standard schedules, Fig. (16), with a continuous check on the on-going operations. The duty list needs to be completed in every detail, changing the location of the sewing head, with the specified elemental tasks.

The existing sewing heads possess several pre-set options. Simplest devices need only to be set for differential or upper fabric transport and for choosing: stitch length, foot pressure, seam speed and thread tension. A cell station (and operator) is actually enabled only for the given on-going production, Fig. (17). The sewing heads for normal stitching 


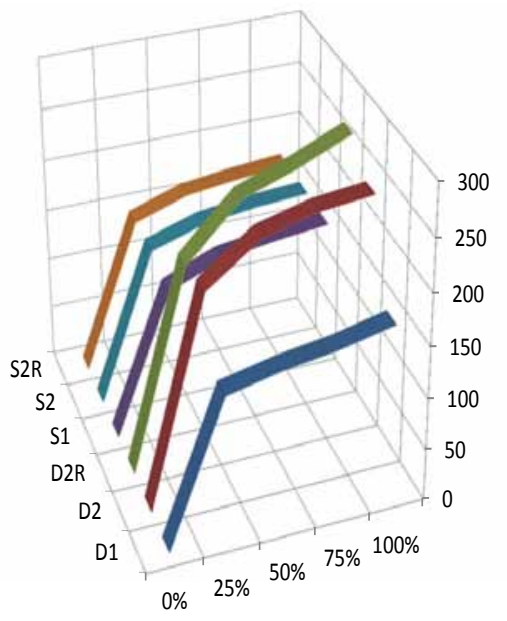

(a)

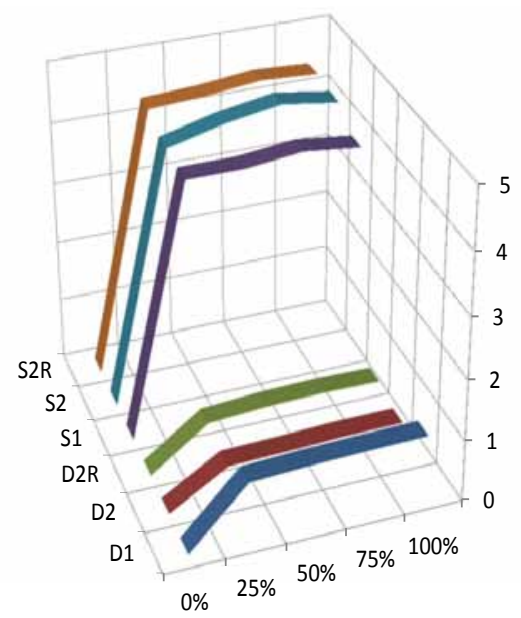

(c)

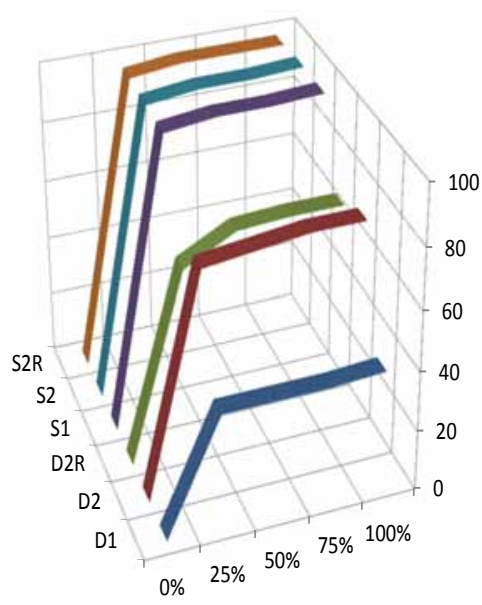

(b)

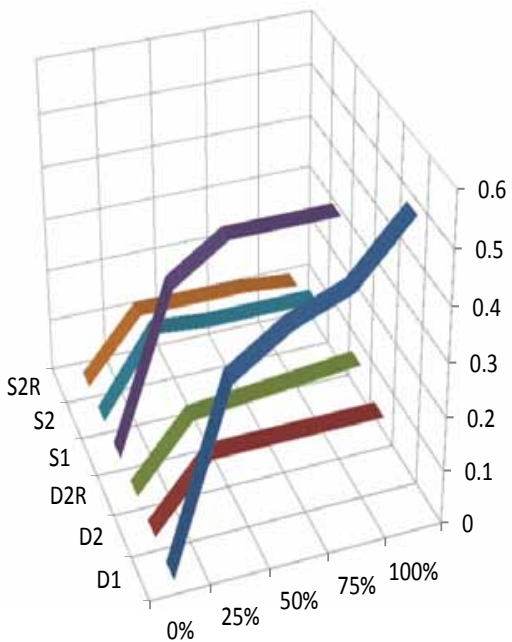

(d)

Fig. (18). Slow AGV deeds: (a) throughput, (b) utilisation ratio, (c/d) in/out average queue.

are, thus, occasionally moved in or out, depending on product type and duty list. Pallet buffering offers a critical benefit, mainly, when a varying product mix is processed and the delivery policy can be optimised to reduce the head reprogramming.

The operator is a critical element; the simulation model needs to deal with random (human-like) job execution times and unexpected stops. The section logistics provides effective means to face flexibility on condition of having inbetween stores and to supply selective dispatching. Pallet operations depend on delivery resources (carrying shuttles and loading/unloading rigs) and on shop information system (a message passing mechanism to specify the work-inprogress, etc). An example study for the underwear manufacturing considers the assembly as composed of three parts by eight seams and finished with waist and leg elastic bands. Cost analysis provides the basic data to weigh the cycle effectiveness. Fixed and variable costs are detailed, including cell set-up, workstation outfitting, sewing heads, local buffering, loading/unloading rig, pallets, shuttles, track layout, etc.; these entities typify by unit investments: operator and supervisor attendance, power supply and maintenance of stations, transporters, etc., overheads and general charges, etc. and also the operating fees must be accurately specified.

Without going too far into details, basic assessments permit comparing productivity measures, observed as average figures over one or more work-shifts, modifying the assembly cycles, front-end sewing units and section logistics. Duplication of sewing heads requires extra supply paths, provided by two shuttles sharing the same track; buffered lay-outs are safer for flexibility issues. The plots of Fig. (18) and Fig. (19) show the cell throughputs with one (S) or two (D) sewing units and different shuttle speeds $(0.5$ or $1.0 \mathrm{~m} / \mathrm{s})$.

The flow balancing is better achieved at the lower speed (unless part assorted picking has prior processing). The results consider this dispatch policy, looking at cell throughput, workstation utilisation ratio and input or output buffering capabilities.

Compared to the previous case examples, the present distributed information system needs to deal explicitly with workers specialisation and adeptness, included in the duty 


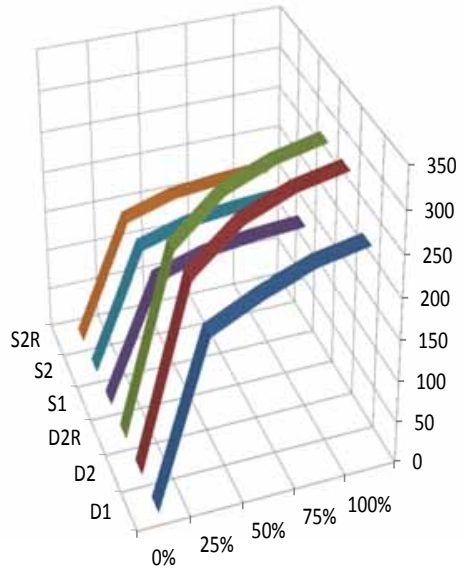

(a)

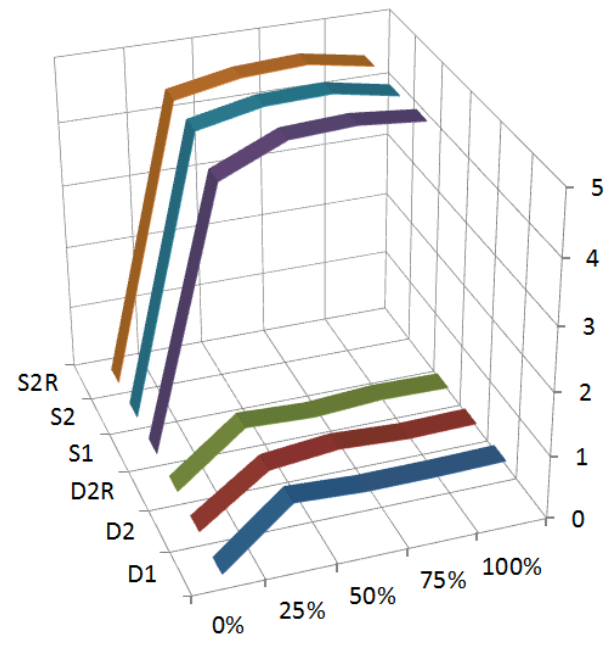

(c)

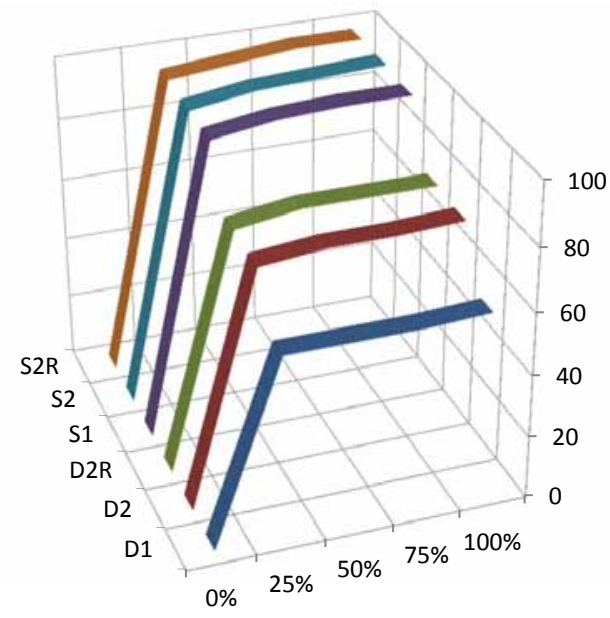

(b)

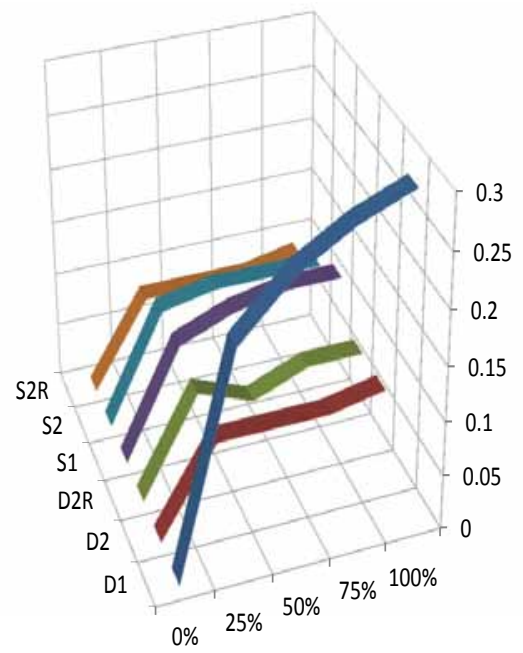

(d)

Fig. (19). Fast AGV deeds: (a) throughput, (b) utilisation ratio, (c/d) in/out average queue.

cycles as front-end operators. In this respect, the shop-floor software requires deeper concern, to make easy the interfacing with low structured environments (and not with thoroughly automatic fixtures) and to support unsteady duty cycles and heuristic decision keeping modes as inherent feature at work-cycles level.

\section{CONCLUDING REMARKS}

The intensive use of robotics and intelligent manufacture is important in competing successfully in the cross-border economy. The survey considers the resort to these tools, within the clothing industry, where low cost labour has especially driven the marketplace, in recent decades, toward productive break-up, with drawbacks on preserving the process control uniformity. Several hints are described for the use of robotic tools in the production flow and management of the related operations. Emphasis is placed on aiming at high quality products in a customised production environment. For the clothing manufacturers of developed countries, the automation concepts presented here offer an alternative (and perhaps the only alternative) to out-sourcing and overseas production.
Intelligent manufacturing is established option, showing the new ideas of the economy of scope after centuries of economy of scale. The option is linked to robot concepts, thus with the idea of removing front-end workers from the material flow lines. The approach is characterising feature of industrialised countries, connected to the availability of hightech rigs, with market-driven production at buyers' satisfaction and with social requests for workers' better lifeconditions. The clothing businesses have taken very little part to the evolution. The facts appear puzzling, at least, for three reasons: the market-share of the textile and garment production; the customised value creation in the fashion segments; the benefits of direct and strong links between users and supply-chain.

The study looks after a structured approach to intelligent manufacturing, based on an integrated management of information flow, while the material one progresses. The effectiveness depends on exploiting flexibility, to reach return on investment by market-driven processes, adapted to grant buyers' satisfaction $[13,14]$. The goal requires timevarying schedules, ruled by on-process govern decision schemes, continuously up-dated by company-wide 
information systems. The criticality, more than on the factory automation, outcomes from the process visibility and knowledge share. To that purpose, the shop data flow instrumentation and the manufacture processes simulation software are standard requirements. The latter makes possible to assess the effects of flexibility on the different (strategic, tactical or execution) ranges, by tests accomplished in virtual reality.

The all matter is tackled with concern of practical requests and fallouts. The general set-ups are properly defined, since they need the merging into the material flow of all the worthy data, so that individual work-tasks are timely modified to grant the business achievements, exploiting the available resources and reliably propagating the forecast quality. The implementation of these ideas leaves, however, open points, particularly, at the instrumental front-end, when investments in high-tech hardware are weighed against improvements in effectiveness. Certainly, the solutions are not unique; every issue is a compromise between competing requests and is made efficient, under specified contexts.

The extended use of distributed intelligence and scope economy techniques are important way, for successfully competing in today's world. The survey considers the use of these tools within the clothing industry, where low cost labour has especially driven the marketplace, recently, toward productive break-up, with drawbacks on preserving the process control uniformity [15]. Several hints are described for the use of modern manufacturing tools in the production flow of clothing and in the management of the related operations. Emphasis is placed on maintaining a high quality product in a customised production environment [16]. The three example applications distinguish each other for front-end automation; the resort to different solutions characterises clothes industry: return on investment cannot always justify hardware innovation. The situation drastically changes, if shop information systems are considered: relevant benefits, all the times, follow from process visibility and on-process adaptation, making possible economy of scope, as the example cases show.

\section{CONFLICT OF INTEREST}

The authors confirm that this article content has no conflicts of interest.

\section{ACKNOWLEDGEMENTS}

Declared none.

\section{REFERENCES}

[1] M. Acar, "Intelligent textile machines and systems", In: Mechatronics Design in Textile Engineering, M. Acar, Ed. Kluwer Acad. Press, USA, 1995, pp. 61-66.

[2] N. A. Hunter, R. E. King, H. L. W. Nuttle, and J. R. Wilson, "North Carolina apparel pipeline modelling project", Intl. J. of Clothing Science and Technology, vol. 5, pp. 19-24. 1993.

[3] D. H. Kincade, "Quick response management system for the apparel industry: definition through technologies", Clothing and Textiles Research J., vol. 13, no. 4, pp. 245-251, 1995.

[4] U. Meyer, "Automation and control in textile production", In: Globalisation: Technological, Economic and Environmental Imperatives - 75th World conference of Textile Institute, 1994.

[5] A. Seyam, and F. Sun, "Manufacturing technology for apparel automation: lay-up module, part I", Intl J. Clothing Sci Technol., vol. 5, no. 3/4, pp. 44-59, 1994.

[6] A. Antonelli, The economics of innovation: critical concepts in economics. Rutledge, New York 2008.

[7] G. J. Olling, G. Jacucci, K. Preiss, and M. Wozny, Eds., Globalisation of manufacturing in the digital communication era: innovation, agility and the virtual enterprise. Kluwer Acad. Pub., Norwell, 1998.

[8] R. C. Michelini, G. M. Acaccia, M. Callegari, R. M. Molfino, and R. P. Razzoli, "Shop controller-and-manager for intelligent manufacturing", In: Management and Control of Manufacturing Systems, S. Tzafestas, Ed. Springer Verlag: London, 1997, pp. 219254.

[9] J. Olhagher, and F. Persson, Eds., Advances in production management system. Springer: Boston 2007.

[10] J. X. Liu., Ed., New developments in robotics research. Nova Sci. Pub: New York, 2005.

[11] R. C. Michelini, Robot age knowledge changeover. Nova Sci. Pub: New York, 2009

[12] S. Zhang, L. Lin, and A. Kumar, Materials characterisation techniques. CRC Press: Abingdon, 2009.

[13] G. L. Kovàcs, P. Bertòk, and G. Heidegger, Eds., Digital enterprise challenge: lifecycle approach to management and production. Kluwer Acad. Pub: Boston, 2002.

[14] F. Y. Phillips, Market-oriented technology management: innovating for profit in entrepreneurial time. Springer: Berlin, 2001.

[15] N. L. Russo, B. Fitzgerald, and J. I. DeGross, Eds. Re-aligning research and practice in information systems development: the social and organizational perspective. Kluwer Academic Pub: New York, 2001.

[16] R. C. Michelini, and R. P. Razzoli, "Robotics in Clothes Manufacture", International Journal of Mechanical Engineering and Applications, vol. 1, no. 1, pp. 17-27, 2013. 